\title{
Incorporating stakeholders' knowledge in group decision-making
}

\author{
Salem Chakhar \\ Portsmouth Business School, University of Portsmouth, Portsmouth, Hampshire, UK. \\ Email: salem.chakhar@port.ac.uk
}

Inès Saad

MIS, University of Picardie Jules Verne, Amiens, France.

France Business School, Amiens, France

Email: ines.saad@u-picardie.fr

Salem Chakhar is with the Portsmouth Business School in the Operations \& Systems Management subject group. Salem Chakhar has a PhD in Computer Science from the University of Paris-Dauphine (Paris, France), an MPhil degree in Computer Science and Modelling from the High School of Management of Tunis (Tunis, Tunisia), and a degree in Computer Science from the Faculty of Economics and Management, University of Sfax (Sfax, Tunisia). He has published in journals such as International Journal of Geographical Information Science; Computers, Environment and Urban Systems; Information Sciences; Information and Software Technology; European Journal of Operational Research; Environment and Planning B: Planning and Design; and Decision Support Systems. Salem Chakhar is a member of the International Cartographic Association (ICA) commission on geospatial analysis and modelling.

Inès Saad is an Associate Professor at France Business School. She is also a Researcher within the MIS Laboratory at the University of Picardie Jules Verne (France). She obtained her Ph.D. in Computer Science from the University of Paris-Dauphine in 2005. From 2005 to 2006, Dr. Saad has been an Assistant Professor at the University of Paris-Dauphine. The focus of her research is on the knowledge management, information system and multiple criteria decision making. She has several publications in international conferences and journals such as DSS, EJOR, GDN, and SIM. 


\section{Incorporating stakeholders' knowledge in group decision-making}

The objective of this paper is to characterize the group decision-making process. Three basic questions should be considered in group decision-making: (i) how to extract stakeholders' knowledge and preferences, (ii) how to combine these preferences and knowledge coherently and (iii) how to conduct discussions and resolve conflicts. To respond to these questions, this paper distinguishes and discusses several elements characterizing the group decision-making process. More specifically, the paper discusses the following topics: techniques for acquiring stakeholders' knowledge, aggregation strategies and rules, preference parameters elicitation techniques, stakeholder weighting and conflict resolution. The paper also includes a brief discussion of several real-world case studies in which the authors of this study participated.

Keywords: group decision-making, knowledge acquisition, aggregation strategy, aggregation rule, preference parameters elicitation, weighting stakeholders, conflict resolution

\section{Introduction}

Decision-making can be regarded as the cognitive process resulting in the selection of a course of action from several alternative scenarios. Decision-making problems range from everyday decisions, such as the selection of an itinerary to go from one place to another, to more complex decisions involving a large number of individuals, associations, or socio-economic groups. Complex decisions generally require the development of formal methods to handle them. Several methods have been proposed in the literature, including cost-benefit analysis, statistical techniques, evolutionary algorithms, multicriteria analysis. The majority of decision-making methods "assume a single decision maker for simplicity" (Munda, 2004) whereas real-world problems requiring decisions naturally imply multiple decision makers with conflicting objectives and distinct value systems. This multiplicity of objectives and value systems derives 
directly from the multidimensional nature of decision problems. The situation is complicated by what Hendriks (1995) calls the "social context" of the decision problem that creates conflicting objectives, reflecting the diversity of interests and concerns of the stakeholders in the decision-making process. Indeed, the identical decision problem is perceived, designed, formalized and modeled differently by people with different perspectives, such as environmentalists, politicians, or economists. Each stakeholder in the decision-making process has a different perception of the decision problem according to his own objectives and concerns.

Group decision-making (also known as collaborative decision-making) is a situation faced when different stakeholders are collectively included in the decisionmaking process. Several authors have recognized the need for group decision-making methods (Belton and Pictet, 1997; Jelassi et al., 1990), and the literature has proposed different methods (e.g., Hatami-Marbini and Tavana, 2011; Macharis et al., 1998; Matsatsinis and Samaras, 2001; Nurmi et al., 1996; Colson, 2000; Jabeur and Martel, 2007b; Ben Khelifa and Martel, 2001; Chen and Cheng, 2010; Cook, 2006; Cil et al., 2005). The majority of these papers assume the existence of a mediator who acts as a third-party member to structure meetings and reach a final decision based on the facts articulated during the discussions.

The objective of this paper is to characterize the group decision-making process. Three basic questions should be considered in group decision-making: (i) how to extract stakeholders' knowledge and preferences, (ii) how to combine these preferences and knowledge coherently and (iii) how to conduct discussions and resolve conflict situations. In this paper, we discuss the following topics: techniques for acquiring stakeholders' knowledge, aggregation strategies, aggregation rules, preference parameters elicitation techniques, weighting stakeholders and conflict resolution. The 
first topic is related to the first question that should be considered in characterizing group decision-making. The last topic corresponds to the third question. The remaining topics are related to the second question regarding the characterization of the group decision-making process. The paper also includes a brief discussion of several realworld case studies in which the authors of this study participated. It is important to mention that this paper is neither a survey nor a comparative study of group decisionmaking. Rather, it represents the authors' point of view based on their personal research and experience. It is also important to mention that the terms "stakeholder", "decision maker" and "expert" are used in various manners in this paper. Indeed, in realworld decision problems, these terms may refer to different individuals or groups of individuals who may or may not be directly involved in the decision-making process.

The rest of the paper is organized as follows: Section 2 discusses different techniques for acquiring stakeholders' knowledge. Section 3 presents the different aggregation strategies. Section 4 summarizes the different aggregation techniques and rules in group decision-making. Section 5 discusses the different methods to elicit preference parameters. Section 6 addresses the weighting of stakeholders. Section 7 discusses conflict resolution. Section 8 discusses several real-world case studies. Section 9 presents the paper's conclusions.

\section{Acquiring experts' knowledge}

The aim of this section is to discuss the different techniques used to extract and represent stakeholders' knowledge and preferences in group decision-making. Several techniques have been proposed in the literature to acquire and represent experts' knowledge. In addition, a multitude of strategies have been advanced to manage certain conflicts in expertise and reflect experts' knowledge. One strategy identifies the expert most competent to acquire and model knowledge. Another strategy aims at focusing 
only on the consensual knowledge of the experts, knowledge on which various experts agree. Other strategies have also been proposed solely to acquire knowledge such as "the strategy of the biggest generalization". This strategy focuses only on the most general knowledge and avoids specific knowledge. In this context, Labidi (1996) advances 2 approaches to acquire knowledge from multiple experts:

Incremental acquisition of knowledge: This technique focuses on first acquiring knowledge from one expert and then progressively refining the knowledge with subsequent experts.

Combination of expertise models: This technique focuses on acquiring knowledge separately from each expert. Next, the knowledge engineer combines the various experts' models to create an integrated model that is based on consensual knowledge combining different points of view.

The "technique of consensual decision-making" proposed in McGraw and Seale (1987) allows identifying the optimal solution to a problem. This technique is based on the vote of a group of experts on a solution identified during, for example, a brainstorming session. This technique comprises several steps:

1. Presentation of the technique to the experts.

2. Identification of the problem and possible solutions presented by the knowledge engineer.

3. A first round of voting: Each expert may choose three solutions with at most two rationales per option. Propositions that receive a low number of rationales are eliminated.

4. A second round of voting: If, after being reduced, the number of solutions 
remains too high, experts proceed to a second round of voting. In this round, each expert must present no more than two options with no more than one rationale per option. Solutions that have less than a certain number of votes are eliminated; a discussion can occur if necessary.

5. Continuity of the voting process until a unanimous option is agreed upon.

6. Finally, the knowledge engineer checks the degree of confidence of everyone regarding the obtained option.

Huseman (1977) proposed the "Approach of the Nominal Group." The experts meet together but work independently. Every expert "silently" creates a certain number of solutions to a problem. Next, the knowledge engineer selects the most pertinent ideas. These ideas are then expanded. A secret vote to prioritize ideas from the best to the worst then occurs. At the end, to reach a consensual solution by experts, other discussions may follow.

The method of "Brainwriting" was proposed by Boy (1988). This strategy gathers knowledge from different experts to produce ideas within a group. It is based on the formalization of the experts' expertise on a precise subject. At the beginning, the subject is described on sheets of paper available in the middle of the table. Each expert takes a sheet, reads it, writes some notes on it and places it back in the middle of the table. Each expert must write his ideas on all of the papers. Next, a collation of the principal ideas is created. The consensus rate is thus calculated by comparison. This method permits the gathering of a great many ideas.

Moreover, other methods of the "logic design" type have been proposed in the literature to model the knowledge used or created in a project and formalize memories of projects. These methods allow the preservation of all information related to decisions 
made by designers on a predefined project. This information concerns the reasons why certain solution options were explored, why some were eliminated, the project context and what occurred during the project.

These methods were first developed to document the decision process in the design field. Today, however, such methods permit the capitalizing of knowledge in a project memory (Dieng et al., 2001). Of these methods, we will deal only with those that utilize applications such as the IBIS and QOC.

The IBIS (Issue-Based Information System) method was proposed by Horst Rittel et al. in the 1970s (Buckingham-Shum and al., 1997) to support communication within designers' groups and formalize decision-making in a framework of design projects.

The IBIS is a process-oriented approach, structured according to the three different steps of the decision-making process: issues (questions), positions (positions) and arguments (arguments). In fact, when a primary question is presented, a participant in the decision-making process takes a certain position not only by suggesting a solution to the issue presented but also by justifying his position using arguments. Other participants, however, present other positions by proposing other solutions to the identical problem and developing other arguments. These arguments can reinforce a position or oppose other positions. During discussion, new issues may appear.

The QOC (Question, Options, Criteria) method supports the decision-making process in a design activity and is also a process-oriented approach. The QOC allows the presentation of different solutions and includes the advantages as well as the drawbacks of each solution. The use of the QOC method requires precisely specifying the problem, whether the idea concerns an issue, an option or a criterion. Then, the idea must be formalized and well-structured to be added to the diagram. 
The different methods previously proposed by knowledge engineering and the "Rational design" methods are quite appropriate for the acquisition and preservation of the knowledge used and produced by a multitude of experts. However, the application of these methods becomes impractical in complex projects (a great many professions and/or experts, collaboration with the external environment involving many partners and suppliers). Moreover, experts must be mobilized to acquire their knowledge and then validate its modeling, which indicates that experts may be diverted for a certain period of time from performing tasks that are of immediate value to the company to accomplish operations of knowledge capitalization that generate value for the long term.

\section{Perspectives aggregation strategies}

Two main strategies that may be used to combine the judgements and perspectives of decision makers in group decision-making are at the input level or the output level (Dias and Climaco, 2000). It is also possible to combine the input and output levels to reduce or avoid their respective shortcomings as will be explained later in this section. In the rest of this section, utilizing $h \geq 2$, we design the number of decision makers.

\section{Input aggregation strategy}

The input level aggregation strategy proceeds as follows (Figure 1.a): (i) an operator $j($. aggregates the individual inputs $S_{i}(i=1, \ldots, h)$ into a collective input $S$; then (ii) an operator $w($.$) yields the final result R$. The aggregation performed by $j($.) may comprise averaging, minimizing a distance measure, voting, etc. The operator $w($.$) may be any$ type of approach that yields the desired result. Previous studies presenting examples of proposals based on this strategy are Cai et al. (2012), Damart et al. (2007), Dias and

Climaco (2000), Greco et al. (2006), Bi and Chen (2007), Jabeur et al. (2004), Saad and Chakhar (2009), Brigui-chtioui and Saad (2011), and Chakhar and Saad (2012). 
The input-oriented aggregation strategy appears to be technically and practically easier to implement. However, this strategy assumes that decision makers can collaborate effectively and that there is little conflict regarding input data.

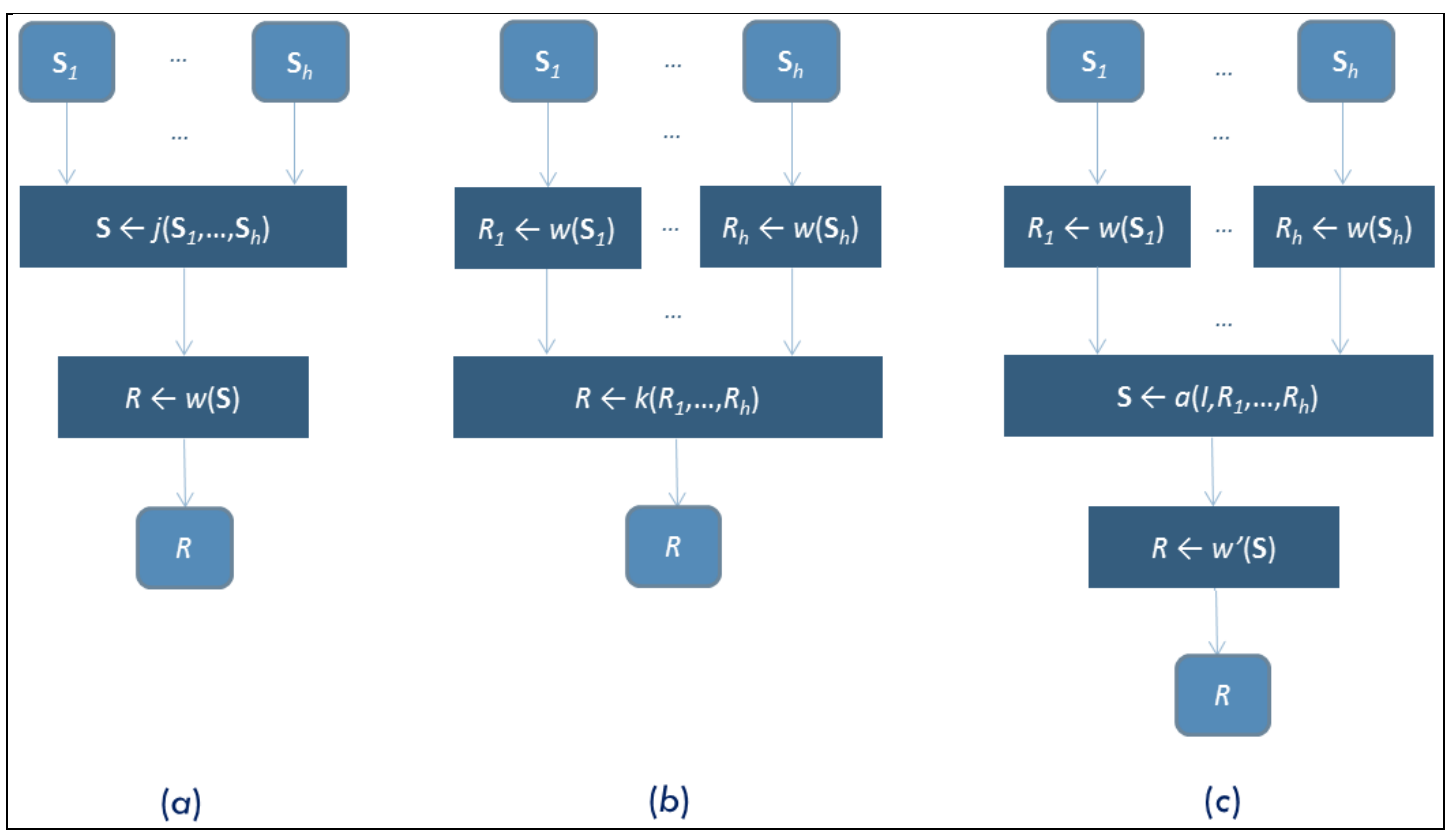

Figure 1. Different aggregation strategies: (a) aggregation at input level, $(b)$ aggregation at output level, $(c)$ mixed input-output aggregation. Note: Diagrams for cases $(a)$ and $(b)$ are inspired by Dias and Climaco (2000).

\section{Output aggregation strategy}

The output level aggregation strategy proceeds as follows (Figure 1.b): (i) the operator $w($.$) yields the individual results R_{i}(i=1, \ldots, h)$, and (ii) an operator $k($.$) aggregates the$ individual results $R_{i}(i=1, \ldots, h)$ into a final result $R$. The operator $w($.$) may be any type$ of approach that yields the desired result. The aggregation performed by $k($.$) may$ comprise averaging, minimizing a distance measure, voting, etc. Previous research includes examples of proposals based on this strategy: Bregar et al. (2008), Greco et al. (2009), Jabeur and Martel (2007b).

The output-oriented strategy appears to be more flexible than the input-oriented strategy because the implied decision makers can work independently. This is an 
important aspect of the decision-making process because it is appropriate for decision makers who are geographically distributed and/or who have time constraints. Furthermore, the output-oriented aggregation strategy appears to be more appropriate for decision situations in which the judgements and objectives of implied participants are conflicting. However, the main shortcoming of the output aggregation strategy is its technical complexity and difficulty of use in practice.

\section{Mixed input-output aggregation strategy}

To take advantage of both aggregation strategies and to avoid or reduce their respective shortcomings, we designed a mixed aggregation strategy that is shown in Figure 1.c. The proposed mixed input-output aggregation strategy proceeds as follows:

(1) An operator $w($.$) yields the individual results R_{i}(\mathrm{i}=1, \ldots, h)$;

(2) an operator $a($.$) uses the common input information I (e.g., common preference$ parameters $)$ and the individual results $R_{i}(i=1, \ldots, h)$ to construct the collective input $S$;

(3) an operator $w^{\prime}($.$) yields the final result R$.

As shown in Figure 1.c, the first phase of the mixed aggregation strategy corresponds to the first phase of the output aggregation strategy, and the third phase of the mixed aggregation strategy corresponds to the second phase of the input aggregation strategy. The second phase of the mixed aggregation strategy combines the first phase of the input strategy and the second phase of the output strategy in the sense that it leads to the collective input $S$ (as in the first phase of the input aggregation strategy) by aggregating the output of individual results $R_{i}(i=1, \ldots, h)$ (as in the second phase of the output aggregation strategy). 
We think that the mixed aggregation strategy combines the advantages of both the input and output aggregation strategies. Particularly, the decision makers can work independently during the first phase; however, all of them should agree on the final result of the third phase. This agreement requires some collaboration between decision makers. The second phase is an automatic phase, and there is no need for intervention from the decision makers.

\section{Aggregation rules}

One of the most important issues in group decision-making is defining appropriate techniques to combine individual preferences. The importance of this issue has been addressed in previous research as in Ben Khelifa and Martel (2001), Cook (2006), Jabeur and Martel (2007a) and Jabeur and Martel (2007b). Ideally, one can define an aggregation rule that reproduces the "totality" of individual preferences. Unfortunately, this is not often possible in practice because decision makers generally have conflicting objectives and preferences.

Three categories of aggregation rules may be distinguished (Azibi and Vanderpooteen, 2002): statistical, functional and rule-based techniques. The main advantages of statistical operators are their compactness and simplicity. The functional aggregation rules are based on using functions, i.e., a weighted-sum or distance measure. Researchers such as Brigui-Chtioui and Saad (2011) and Jabeur and Martel (2007b) have presented examples of proposals based on functional aggregation. Rule-based aggregation techniques are based on using Boolean and/or "IF...,THEN..." rules. These rules apply to complex situations for which statistical or functional aggregation rules cannot be applied (Azibi and Vanderpooten, 2002). An example of rule-based aggregation techniques use is presented in Azibi and Vanderpooten (2002).

In some situations, aggregating the input data is simply based on discussion among different decision makers as in Damart et al (2007), Saad et al. (2005) and 
Mercat-Rommens et al. (2010). In the latter paper, for instance, although several decision makers have been implied, the collective input has been defined by the responsible project manager based on her expertise and the information presented by different decision makers.

The aggregation rule should permit reaching consensus by considering the points of view of decision makers that are favorable and those that are not. The majority rule, which is characterized by its simplicity, anonymity and neutrality and its low demand in terms of computational time, appears to be an excellent candidate for the aggregation mechanism. There are different manners in which to implement the majority/veto principle. Generally speaking, the majority/veto principle requires two conditions: (i) there is a "sufficient" majority of decision makers who are favorable to a given decision; and (ii) when the first condition holds, none of the minority of decision makers presents an "important" opposition to this decision.

Let us assume that the decision problem concerns the assignment of decision objects to different decision classes, $C l_{1}, \ldots, C l_{n}$. These decision classes may, for instance, represent different levels of risk as in Chakhar and Saad (2012). The above conditions can be implemented as follows: An object $x$ is assigned to a class $C l_{t}$ if and only if (i) at least $\lambda=50 \%$ (any other value in the range of 50\%-100\% may also apply) of decision makers agree with this decision and (ii) at most $\theta=25 \%$ (any other percentage in the range of 0-49\% may also apply) of the minority of decision makers oppose this decision (i.e., assignment of $x$ a class $C l_{t}$ ). This idea has been applied in Chakhar and Saad (2012).

\section{Preference parameters elicitation approach}

In decision-making, decision makers are often called upon to specify parameters 
required by the aggregation rule such as the weight of various criteria. This remains the case with group decision-making. We distinguish two major approaches to specifying preference parameters (Dias and Climaco, 2000): direct or indirect. It is also possible to combine these two strategies.

The direct elicitation approach is the most used in practice. In this approach, decision makers explicitly specify the values for all preference parameters. This is not an obvious exercise in practice, especially in group decision-making. Indeed, specifying these parameters requires a significant cognitive effort from the experts. In addition, in this approach we assume that all implied experts understand the meaning and role of these parameters and that all of them agree on the values of these parameters.

In the indirect elicitation approach, parameter values are implicitly obtained. The basic idea of this approach is to deduce values for preferred parameters from holistic information supplied by the decision makers. A well-known approach to indirect elicitation is the case-based reasoning method. Some authors design the casebased reasoning approach by aggregation/disaggregation as in Dias et al. (2002). Casebased reasoning can be structured in three stages: (i) definition of the holistic information, (ii) inference of preference values, and (iii) use of inferred values. The objective of the first stage is to specify the holistic information. Holistic information is global judgements on decision objects and thus represents aggregated information. The objective of the second stage is to infer the values of preferred parameters using the holistic information as input. The values of inferred parameters are obtained by disaggregating the global information provided by the decision makers. At the end of this stage, the decision makers should agree on the inferred values. Otherwise, the two first stages can be repeated using different input data. The objective of the third stage is 
to use the obtained results to apply the aggregation rule. This approach has been used in different real-world decision problems, e.g., Chakhar and Saad, 2012; Damart et al., 2007; Guay et al., 2011; Mercat-Rommens et al. 2010.

The case-based reasoning approach has two main advantages. First, it substantially reduces computing time. Second, case-based reasoning reduces the cognitive effort required from the experts. This second advantage is particularly important in practice. Indeed, we think that experts are more cooperative in producing assignment examples than giving exact values for the different parameters. This fact has been confirmed by real-world decision problems (see Chakhar et al., 2012; Guay et al., 2011).

The direct and indirect elicitation approaches can also be used jointly as in Mercat-Rommens et al. (2010). The basic idea of a mixed direct-indirect elicitation approach is to fix the values of certain parameters and to infer the values of others. The process is repeated several times to ensure the stability of the inferred values. The main argument for using the mixed approach is the substantial gain in terms of computing time compared to the use of the indirect approach alone and the relatively low cognitive effort compared with the use of the direct elicitation approach alone.

In spite of the attractiveness of case-based reasoning, one should mention that the quality of the output depends largely on the quality of the input.

\section{Weighting stakeholders}

In group decision-making, it is often assumed that the decision makers have different "powers" or "weight". This fact is recognized by different authors, including Cook (2006), Jabeur and Martel (2007a), and Jabeur and Martel (20007b). We may distinguish several manners in which to define these weights: 
1) weights are defined explicitly by a mediator or an external person;

2) weights are defined based on the hierarchical levels of implied decision makers;

3) weights are defined explicitly using a given method;

4) weights are defined implicitly based on input.

Although the first method appears to be quite subjective, it may be useful in practice, especially when the expertise and neutrality of the mediator is well recognized by the different implied decision makers. An example of using this method is provided by Leyva-Lopez and Fernandez (2003). In the second case, weight generally reflects the hierarchical levels of decision makers in the organization. This method can be justified in some decision situations, especially when one or some of the decision makers are financially involved and the final decision has serious consequences for their investment. In addition to the usefulness of the two first cases in practice, there is a need for more formal and objective methods, as indicated by Cook (2006). Researchers such as Ramanathan and Ganesh (1994) thus propose using the Analytic Hierarchy Process (AHP) (Saaty, 1980) method to derive members' weight. Martel and Ben Khelifa (2000) proposed a method of using individual outranking indices. Jabeur et al. (2004) proposed using a previous method (Zeleny, 1982) to integrate the relative importance of the groups' members into the consensus construction.

In some situations, the weight is not used as in Saad and Chakhar (2009) and Greco et al. (2006). However, this may be perceived as an implicit use of weight in the sense that all decision makers are assumed to have identical power.

We think that the most important characteristic of weight definition methods is the objectiveness of weight. In this respect, Cook (2006) advocated that the question is 
not how to use weight, but rather how to objectively quantify weight. In Chakhar and Saad (2012), for instance, the contribution of each decision maker to the collective decision is measured by the quality of input data provided by the decision maker. We think this a more objective manner than more conventional methods. Indeed, generally, more experienced decision makers provide input data and knowledge of higher quality. This leads to more "democratic" decisions because decision makers are only differentiated based on their level of "expertise" and not on their hierarchical levels. This is confirmed in different real-world applications in which the authors were engaged; see Saad et al. (2005), Saad and Chakhar (2009) and Mercat-Rommens et al. (2010).

\section{Conflict resolution}

Supporting conflict resolution is an important characteristic of group decision-making. In some proposals, conflict resolution is simply addressed by discussion. This is the case with Damart et al. (2007) and Saad et al. (2005). Some proposals use advanced tools to resolve the conflict between different decision makers. For instance, BriguiChtioui and Saad (2011) proposed to use multi-agent models to address conflict situations. Another example is the ELECTRE-GD method proposed by Leyva-Lopez and Fernandez (2003). This method contains two phases. The objective of the first phase is to solve the conflict between the information provided by individual rankings and fuzzy relations among preferences. The objective of the second phase is to define a fuzzy relation that is highly ranked for a Supra Decision Maker, which should be exploited in some rational manner to derive a final group ranking. Finally, some proposals do not support any conflict resolution such as in Bi and Chen (2007), Chakhar and Saad (2012) and Greco et al. (2006). 


\section{Case studies}

The objective of this section is to discuss some real-world case studies in which the authors have been implied. The description of these cases in respect to the discussed characteristics of group decision making is summarized in Table 1. Further information can be found in the given references.

\begin{tabular}{|l|l|l|l|}
\hline & $\begin{array}{l}\text { Case study 1: } \\
\text { Management of } \\
\text { post-accident } \\
\text { nuclear risk }\end{array}$ & $\begin{array}{l}\text { Case study 2: Heat } \\
\text { islands exposition } \\
\text { in the QMC }\end{array}$ & $\begin{array}{l}\text { Case study 3: } \\
\text { Management of } \\
\text { company's crucial } \\
\text { knowledge }\end{array}$ \\
\hline $\begin{array}{l}\text { Knowledge } \\
\text { acquiring }\end{array}$ & Brainstorming & Brainstorming & Brainstorming \\
\hline $\begin{array}{l}\text { Aggregation } \\
\text { strategy }\end{array}$ & Input & Mixed & Input \\
\hline Aggregation rule & $\begin{array}{l}\text { Informally by } \\
\text { project responsible }\end{array}$ & $\begin{array}{l}\text { Majority/veto and } \\
\text { rules }\end{array}$ & By discussion \\
\hline $\begin{array}{l}\text { Preference } \\
\text { parameters } \\
\text { elicitation }\end{array}$ & $\begin{array}{l}\text { Indirectly by } \\
\text { inference }\end{array}$ & Not required & Not required \\
\hline $\begin{array}{l}\text { Stakeholders } \\
\text { weighting }\end{array}$ & Not used & Not required & Not required \\
\hline $\begin{array}{l}\text { Conflict } \\
\text { resolution. }\end{array}$ & By discussion & By discussion & By discussion \\
\hline
\end{tabular}

\section{Management of post-accident nuclear risk}

The PRIME project considered here concerns the management of post-accident nuclear risk in southern France. A full description of the project is available in MercatRommens et al. (2010). The objective of PRIME was for the decision makers, the stakeholders and representatives of the territory, to jointly develop a multicriteria evaluation approach to analyze and characterize the contaminated territory. This evaluation would be utilized individually by the managers of the risk. The evaluation approach would allocate to each district of the study area a degree of weight representing the risk to the district of a nuclear accident resulting in contaminants released into the atmosphere. 
The PRIME project was a stimulating project and an excellent opportunity to apply decision-making tools in practice and to discuss issues and meet with different stakeholders who naturally have different objectives and preferences. The project was also an important occasion on which to address and discuss different conceptual and technical problems. One of the most important problems encountered during the PRIME project concerned the definition of preference parameters. In the PRIME project, the multicriteria classification method ELECTRE TRI (Figueira et al., 2005) was used for different simulations. During PRIME, preference parameters (especially criteria weight) required by ELECTRE TRI were inferred using the IRIS software (Dias and Mousseau, 2003), which supports the aggregation/disaggregation approach proposed in Dias et al. (2002).

The second thoroughly discussed issue during the PRIME project was the manner in which the preference information of the different implied stakeholders was incorporated into the decision-making process. The aggregation/disaggregation approach of Dias et al. (2002) is designed for single decision makers and thus could not be applied in this case. For simulation purposes, the project manager responsible informally aggregated the assignment examples provided by the decision makers, and then the aggregation/disaggregation approach of Dias et al. (2002) was used.

\section{Heat islands exposition in the QMC}

This case study was conducted as part of an action-research project whose purpose was to strengthen the resilience of the Québec Metropolitan Community (QMC, Québec, Canada) to climate change (Dubois et al., 2012). We used a methodology of four phases. The objective of the first phase was to assess and structure the input data. It involved the identification and assessment of evaluation factors and the definition of a risk scale. In this particular application, a list of seven factors was used. Next, the 
experts jointly designed an ordinal relative risk scale of six levels from the lower relative risk (1) to the highest relative risk (6) to compare areas in the QMC. In the second phase, each team (i) identified a set of learning examples and (ii) used the Dominance Rough Set Approach (DRSA) (Greco et al., 2001) to approximate and summarize the information provided by the experts. The objective of the third phase was to combine the output of the previous phase and then infer a set of collective decision rules. The fourth phase utilized the inferred decision rules to classify all sectors of the QMC.

In this application, the result obtained by the aggregation procedure and the results directly and jointly specified by the experts during data collection sessions coincide with approximately $65 \%$ of the decision objects. One of the practical problems faced during this application was the lack of information. Indeed, initially a large number of attributes were identified by the experts. However, only seven attributes for which data were available for all the districts of the QMC were used to produce final risk maps. Another problem concerned the quality of information. In fact, the resolution of the data used was $250 \times 250 \mathrm{~m}$. The effect of low resolution explains the observed difference between temperature maps executed by the National Institute of Public Health of Quebec and the maps obtained by the proposed risk exposition approach. Furthermore, although the aggregation procedure requires the definition of several parameters, experts may not agree on these parameters' values. A possible solution to this problem could be the use of an indirect elicitation approach.

\section{Management of company's crucial knowledge}

Given the high cost of the operations of capitalization as well as limited resources, a French car manufacturer must focus the investment he intended to make on the knowledge that he deemed "unavoidable knowledge" (tacit and explicit) to render it 
accessible to those who need it. It is a question, for him, of not losing touch with this type of knowledge, to preserve the feedback on experiments on the projects and to transfer this knowledge to less experienced engineers. Thus, the problem of the car manufacturer was to determine the crucial knowledge mobilized and produced during the realization of the development projects of the car products. In this context, we have proposed a multicriteria method to enable those in charge of the projects to present arguments regarding what knowledge should be preserved and shared. Moreover, the choice of this knowledge results from a process of collective decision making established by a combination of multiple project deciders' viewpoints.

Thus, we have based our method on the use of models and tools reserved for the modelling of the processes for the acquisition of knowledge held by the experts to identify knowledge (potential actions) and built criteria.

Depending on the evaluations of knowledge using the different criteria specified by the deciders, the mediator asks each decision maker to assign the "reference knowledge" to only one of the following ordered decision classes: Cl1: decision class of "non-crucial knowledge", corresponding to knowledge whose inclusion has been shown to be unnecessary; $\mathrm{Cl2}$ : decision class of "crucial knowledge", corresponding to knowledge shown to be necessary for inclusion.

The decision is to assign knowledge to the decision classes of "crucial" or "noncrucial" knowledge. Hence, we obtain a number of decision tables that equal that of the decision makers. Each decision table contains the values $\mathrm{F}(\mathrm{Ki}, \mathrm{gj})$ corresponding to the evaluation of each knowledge $\mathrm{Ki}$ by each criterion gj as well as its association with either decision class.

The decision table corresponding to a decider comprises 32 lines and 15 columns. Each line is associated with one of the 32 pieces of "reference knowledge" 
that we want to order and is characterized by 15 criteria. The last column is reserved for the decision made by the decider concerning each piece of "reference knowledge". From the decision tables of each decider (expert), the mediator has used one of the inference algorithms (DOMLEM, Explore) proposed in the DRSA method to infer for each decider the decision rules consistent with his allocation examples. The mediator analyzes, with each decider, the set of rules inferred from examples presented by each of the deciders. The mediator first determines whether the rules show some defect. These defects may originate from (i) the hesitation of the decider while allocating knowledge in the decision class; (ii) the change in the deciders' viewpoints during the decision process. During experiments in the field, we noticed that the decider can change his mind concerning the evaluation of knowledge over a criterion (depending, for instance, on the validity of his knowledge). (iii) the inconsistency of the criteria (a missing criterion, an extra criterion, etc.)

Once the origin of the defect is identified, the mediator corrects it with the decider. He proceeds incrementally because defects are identified in the rules base and the decider intends to modify the allocation examples and/or the criteria. This iterative process allows a better understanding of the decision rules chosen by each decider. In addition, the analysis of these rules can be conducted during individual meetings of an average duration of 2 hours. For each decider, two types of decision rules are determined: those associated with knowledge belonging certainly to the decision class of "crucial knowledge" and those rules concerning knowledge that belongs to the "crucial knowledge" class. Among these rules, the presider must only retain the "reference knowledge" clearly belonging to the decision class of "crucial knowledge". After analyzing the different inferred rules, a set of rules is maintained for each decider. 
The mediator has defined a unique set of rules corresponding to a set of rules collectively accepted by the deciders using decision rules retained for each one of them. The construction of this set is conducted using a constructive approach based on Belton and Pictet's (1997) work. In effect, we have used the comparison technique proposed by Belton and Pictet (1997). Thus, the mediator has helped deciders determine a set of decision rules that are collectively accepted and based on the different rules retained by each one of them. The quality of these rules has been determined while testing them on examples of allocations of new knowledge by the same deciders.

\section{Conclusions}

In this paper, the authors provided a personal view of acquiring knowledge in group decision-making. The paper discusses several points that are directly related to three basic questions in group decision-making: (i) extracting stakeholders' knowledge and preferences, (ii) combining these preferences and knowledge and (iii) resolution of conflict. The second question has been addressed in terms of aggregation strategies and rules, preference parameters elicitation techniques and weighting of stakeholders. The paper also includes a brief discussion of several real-world case studies in which the authors of this study participated.

This paper may serve as a departure point for a survey and review paper. It will also be interesting to compare the different methods and approaches discussed more formally and in further detail.

\section{References}

Azibi and Vanderpooten, D. (2002). Construction of rule-based assignment models. European Journal of Operation Research, 138(2): 274-293. 
Belton, V. and Pictet, J. (1997). A framework for group decision using a MCDA model: sharing, aggregation or comparing individual information. Journal of Decision Systems, 6(3):283-303, 1997.

Ben Khélifa, S. and Martel, J.-M. (2001). A distance-based collective weak ordering. Group Decision and Negotiation, 10(4):317-329.

Bi, W.-J. and Chen. X.-H. (2007). An extended dominance-based rough set approach to group decision. In Proceedings of the International Conference on Wireless Communications, Networking and Mobile Computing (WiCom 2007), pages 57535756, Shanghai, China, 21-25 Sept 2007.

Boy, G. (1988). Assistance à l'opérateur, une approche de l'intelligence artificielle. Editions Teknea.

Bregar, A., Gyorkos, J., and Juric, M.B. (2008). Interactive aggregation/ disaggregation dichotomic sorting procedure for group decision analysis based on the threshold model. Informatica, 19(2):161-190.

Brigui-Chtioui, I., and Saad, I. (2011). A multi-agent approach for collective decision making in knowledge management. Group Decision and Negotiation, 20(1):19-37. Buckingham-Shum , S., MacLean, A. Bellotti, V. and Hammond, N. (1997). Graphical Argumentation and Design Cognition. Human computer interaction.

Cai, F.-L., Liao, X., and. Wang, K.-L. (2012). An interactive sorting approach based on the assignment examples of multiple decision makers with different priorities, Annals of Operations Research, 197(1), 87-108.

Chakhar, S. and Saad, I. (2012). Dominance-based rough set approach for groups in multicriteria classification. Decision Support Systems, 54(1):372-380.

Chen, Y.-L. and Cheng, L.-C. (2010). An approach to group ranking decisions in a dynamic environment. Decision Support Systems, 48(4):622-634.

Cil, I., Alpturk, O. and Yazgan, H.R. (2005). A new collaborative system framework based on a multiple perspective approach: InteliTeam. Decision Support Systems, 39(4):619-641.

Colson, G. (2000). The OR's prize winner and the software ARGOS: How a multijudge and multicriteria ranking GDSS helps a jury to attribute a scientific award. Computers and Operations Research, 27:741-755.

Cook, W.D. (2006). Distance-based and ad hoc consensus model in ordinal preference ranking with intensity of preference. European Journal of Operational Research, 172(2):369-385, 2006.

Damart, S., Dias, L.C. and Mousseau, V. (2007). Supporting groups in sorting decisions: Methodology and use of a multicriteria aggregation/disaggregation DSS. Decision Support Systems, 43(4):1464-1475.

Dias, L.C., and Climaco, J. N. (2000). ELECTRE TRI for groups with imprecise information on parameter values. Group Decision and Negotiation, 9:355-377. 
Dias, L., Mousseau, V., Figueira, J., Climaco, J. (2002). An aggregation/disaggregation approach to obtain robust conclusions with ELECTRE TRI. European Journal of Operational Research 138: 332-348.

Dias, L., Mousseau, V.(2003). IRIS: A DSS for multiple criteria sorting problems, Journal of Multi-Criteria Decision Analysis, vol. 12, pp. 285-298.

Dubois, C., Bergeron, O., Potvin, A., Adolphe, L. (2012) Adapting cities to climate change: heat and urban form. In: The 8th International Conference on Urban Climates (ICUC 8), 6th-10th August, 2012, UCD, Dublin, Ireland.

Figueira, J.R., Mousseau, V., and Roy, B.(2005). Electre methods. In J.R. Figueira, S. Greco, and M. Ehrgott, editors, Multiple criteria decision analysis: State of the art surveys, pages 133-162. Springer-Verlag, New York.

Greco, S. Mousseau, V. and Slowinski, R. (2009). The possible and the necessary for multiple criteria group decision. In Proceedings of the 1st International Conference on Algorithmic Decision Theory, ADT '09, pages 203-214.

Greco, S., Matarazzo, B. and Slowinski, R. (2006). Dominance-based rough setapproach to decision involving multiple decision makers. In S. Greco, Y. Hata, S. Hirano, M. Inuiguchi, S. Miyamoto, H.S. Nguyen, and R. Slowinski, editors, Proceedings of the 5th International Conference Rough Sets and Current Trends in Computing, Kobe, Japan, November 6-8, volume 4259 of LNAI, pages 306-317. Springer-Verlag, Berlin Heidelberg.

Guay, G., Joerin, F., Chakhar, S. Villeneuve, P. and Lavoie, C. (2011). FLORAIDE: a new decision-making tool for weed species. The 2nd World Conference on Biological Invasions and Ecosystem Functioning Group, Mar del Plata, Argentina, 21-24 November.

Hendriks, P. (1995). Collaborative exploration of spatial problems. In The 7th Collaborative Spatial Decision Making Research Initiative, NCGIA, Santa Barbara, USA, 16-19 June.

Huseman, R.C. (1977). The Role of the Nominal Group in Small Group Communication. In R.C. Huseman, D.M. Logue et D.L. Communication (ed.), Readings in international and Organizational Communication, Hollbrook Press, Boston, pp. 493507.

Jabeur, K. Martel, J. and Ben Khelifa, S. (2004). A distance-based collective preorder integrating the relative importance of the groups members. Group Decision and Negotiation, 13:327-349.

Jabeur, K. and Martel, J.-M. (2007a). A collective choice method based on individual preferences relational systems. European Journal of Operational Research, 177(3):1549-1565.

Jabeur, K. and Martel, J.-M. (2007b). An ordinal sorting method for group decisionmaking. European Journal of Operational Research, 180:1272-1289.

Jelassi, T. Kersten, G.E. and Zionts, S. (1990). Readings in Multiple Criteria Decision Aid, chapter An introduction to group decision and negotiation support, pages 537-568. Springer-Verlag, Heidelberg. 
Labidi, S. (1996). Supporting Inter-Experts Cooperation in Knowledge Acquisition Stage. In COOP Group (ed.), Proceedings of the second International Conference on Cooperative Systems (COOP'96), Juan-les-pins, 12-14 June 1996, INRIA.

Leyva-Lopez, J.C. and Fernandez-Gonzalez, E. (2003). A new method for group decision support based on ELECTRE III methodology. European Journal of Operational Research, 148:14-27.

Macharis, C. Brans, J.P. and Mareschal, B. (1998). The GDSS PROMETHEE procedure - A PROMETHEE-GAIA based procedure for group decision support. Journal of Decision Systems, 7:283-307.

Matsatsinis, N.F. and Samaras, A.P. (2001). MCDA and preference disaggregation in group Decision Support Systems. European Journal of Operational Research, 130:414429.

McGraw, K.L., and Seale, M.R. (1987). Multiple experts knowledge acquisition methodology MEKAM. Proceedings of the 3rd Australian Conference on Zxpert Systems, 1987, pp. 165-197.

Mercat-Rommens, C. Chakhar, S. Chojnacki, E. and Mousseau, V. (2010). Coupling GIS and Multi-criteria Modelling to support post-accident nuclear risk evaluation: an application in the southern France region. LGI, Ecole Centrale Paris, France, Research Report CR-LGI-201018, 2010, http://www.lgi.ecp.fr/Biblio/PDF/CR-LGI-2010-18.pdf.

Munda, G. (2004). Social multi-criteria evaluation (SMCE): Methodological foundations and operational consequences. European Journal of Operational Research, 158(3):662-77.

Nurmi, H., Kacprzyk, J. and Fedrizzi, M. (1996). Probabilistic, fuzzy and rough concepts in social choice. European Journal of Operational Research, 95(2):264-277.

Ramanathan, R. and Ganesh, L. (1994). Group preference aggregation methods employed in AHP: An evaluation and an intrinsic process for deriving members' weights. European Journal of Operation Research, 79(1):249-265.

Saad, I. and Chakhar, S. (2009). A decision support for identifying crucial knowledge requiring capitalizing operation. European Journal of Operational Research, 195(3):889-904.

Saad, I., Rosenthal-Sabroux, C. and Grundstein, M. (2005). Improving the decision making process in the design project by capitalizing on company's crucial knowledge. Group Decision and Negotiation, 14(2):131-145. 